\title{
Towards Designerly Data Donation
}

\author{
Alejandra Gomez Ortega \\ Delft University of Technology \\ Delft, The Netherlands \\ a.gomezortega@tudelft.nl
}

\author{
Jacky Bourgeois \\ Delft University of Technology \\ Delft, The Netherlands \\ j.bourgeois@tudelft.nl
}

\author{
Gerd Kortuem \\ Delft University of Technology \\ Delft, The Netherlands \\ g.w.kortuem@tudelft.nl
}

\begin{abstract}
In-the-wild research allows the HCI community to gain insights into personal behaviour and characteristics. For designers and researchers, this means having access to rich spatiotemporal insights reflecting user's characteristics, behaviours, and needs. However, designerly contexts require contextualized and meaningful data, and collecting it in-the-wild involves a great effort. In addition, ethical implications need to be considered. In this paper, we propose designerly data donation, a participatory approach for data collection in-the-wild, as an effective and ethical way to enable data-centric design processes. We present the potential benefits of designerly data donation around three axes: value gain, data contextualization, and roles and relationships. And we introduce the challenges of designerly data donation at the intersection of HCI, UbiComp, and design.
\end{abstract}

\section{CCS CONCEPTS}

- Human-centered computing $\rightarrow \mathrm{HCI}$ theory, concepts and models.

\section{KEYWORDS}

Data Donation; Personal Data; Data-Centric Design;

\section{ACM Reference Format:}

Alejandra Gomez Ortega, Jacky Bourgeois, and Gerd Kortuem. 2021. Towards Designerly Data Donation. In Adjunct Proceedings of the 2021 ACM International foint Conference on Pervasive and Ubiquitous Computing and Proceedings of the 2021 ACM International Symposium on Wearable Computers (UbiComp-ISWC '21 Adjunct), September 21-26, 2021, Virtual, USA. ACM, New York, NY, USA, 6 pages. https://doi.org/10.1145/3460418.3479362

\section{INTRODUCTION}

The ubiquity of digital technology presents an opportunity to surface subtle yet essential insights from people's lives. Fitness trackers (e.g. Fitbit, Apple Watch, Garmin) and their ecosystems illustrate this richness. First, they prompt users for personal information via self-reports such as age, gender, weight, and height. Then, they collect machine data, meaning data collected, generated, or processed by sensors or algorithms, such as movement, location, sleep patterns, and heart rate. Finally, they branch out to other applications, where they further integrate insights including calendar, caloric input, mood, menstrual cycle and related symptoms. This

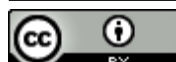

This work is licensed under a Creative Commons Attribution International 4.0 License.

UbiComp-ISWC '21 Adjunct, September 21-26, 2021, Virtual, USA

(C) 2021 Copyright held by the owner/author(s)

ACM ISBN 978-1-4503-8461-2/21/09.

https://doi.org/10.1145/3460418.3479362 multidimensional dataset could portrait a rich spatiotemporal representation of a person's behaviour and characteristics [Gabriele and Chiasson 2020] with the potential to unlock a deep understanding of how people navigate through physical and digital spaces and experiences.

In recent years, the HCI community 'turned to the wild' [Chamberlain and Crabtree 2020] to better understand the 'unremarkable' of everyday life [Crabtree et al. 2020]. By deploying sensorized probes, conducting experience sampling studies as well as leveraging existing data, and digital technologies to gain the necessary insights into understanding people's behaviours and characteristics [Crabtree et al. 2020]. Design researchers, entrusted with understanding people's needs, also find ways to incorporate this into the design process. Through sensorized probes, prototypes, and connected products and services with which people interact daily (e.g. smartphone apps, voice assistants, connected appliances). For them, these new types of data provide valuable insights unavailable with traditional data collection methods, including how design solutions are used in-the-wild, and who are their users [Speed and Oberlander 2016].

However, there is a gap between the abundant production of data from connected products and services - hereinafter machine data - and the effort required to collect contextualised and meaningful thick data for design researchers and practitioners. Since contextualization, depicting the nuances of the data (e.g., what, who, where, how, why), currently requires intimate interventions, which are often not scalable [Bogers et al. 2016; Bourgeois et al. 2014a; Chang et al. 2017]. As a result, design researchers and practitioners face several challenges which turn these opportunities into utopian visions [Dove et al. 2017; Gorkovenko et al. 2020]. In addition, ethical implications emerge as the data is purposefully intimate: the aim is to understand people's behaviours in-depth to surface subtle but critical insights. In this paper, we propose to leverage data donation - an existing concept from the medical field - as an effective and ethical way to enable data-centric approaches in designerly contexts. First, Section 2 depicts the current use and limitation of machine data in design and introduces data donation from the health research. Then, Section 3 presents the potential benefits of designerly data donation in design around three axes: value gain, data contextualisation, and roles and relationships. Finally, Section 4 lays out critical research questions to address the designerly data donation challenges at the intersection of HCI, UbiComp and design.

\section{BACKGROUND}

\subsection{Data in Design}

The design process is inherently data-centric; for decades, design researchers and practitioners have relied on qualitative data (e.g., 
observations, interviews, focus groups) to inform their understanding of the user needs and behaviors [Salvador et al. 2020]. Today, as processing power is continuously increasing and ubiquitous computing is ever more present in products and services, they are introducing a different type of data into the design process, machine data (i.e., data collected, generated, or processed, by sensors or algorithms). Examples of products and services incorporating machine data include lightbulbs, vacuum cleaners, and cars. These products are able to communicate with users, manufacturers, and even other products [Giaccardi et al. 2016; Speed and Oberlander 2016].

Design researchers and practitioners have adopted machine data as a new design material with the potential to enable a rich and detailed understanding about the user needs, preferences, behavior and traits [Speed and Oberlander 2016; Verganti et al. 2020]. Consequently, machine data is being used to inform the early stages of the design process, where it enriches traditional design research methods, such as design ethnography [Chang et al. 2017; Giaccardi et al. 2016] and experience sampling [Van Berkel et al. 2017], and gives rise to other methods such as technology probes [Hutchinson et al. 2003; Worthy et al. 2016], data-enabled design [Bogers et al. 2016, 2018], and participatory data analysis [Bourgeois et al. 2014a,b]. Furthermore, since machine data can be embedded into products and services, it is also being used to remotely understand user behaviors from real-use insights in-the-wild and to optimize, or customize design solutions [Churchill 2018; Verganti et al. 2020]. Machine data presents an opportunity to understand people, their context, experiences, and interaction with other people, products and services in-the-wild. Thus, it could be used to enrich the daily lives of individuals and communities.

In practice, there are a few hurdles to overcome for machine data to be accessible and valuable for design researchers and practitioners. First, machine data collection can be burdensome. It is often expensive, and time-consuming, given that requires scalable sensorization (i.e., building data-driven probes and prototypes) and close collaboration with computer scientist or data scientist [Dove et al. 2017; Gorkovenko et al. 2020], and it is subject to regulatory frameworks such as the GDPR that might complicate and restrict it [Bourgeois et al. 2018; Gorkovenko et al. 2020]. Second, people might be reluctant to share machine data, often containing highly personal information, since it could expose them to privacy and security violations [Gabriele and Chiasson 2020; Goodman 2014]. Third, machine data is often decontextualized, and lacks the rich and meaningful details that make it valuable for designerly contexts [Bornakke and Due 2018; Gorkovenko et al. 2020]. Facing these challenges is critical to enabling a future where people have meaningful physical and digital experiences by design. Hence, we turn our focus to data donation, an alternative to data collection from people, with people, existing in the medical field.

\subsection{Data Donation}

Data donation is the process by which a person (i.e., data donor) transfers their personal data, without expecting anything in return, to another person or entity (i.e., data receiver) who will use this data in a given way in a given context [Prainsack 2019; Skatova and Goulding 2019]. Through data donation, an alternative to personal data collection, people are invited to exercise their autonomy and sovereignty by granting control over their data and deciding where it goes, who has access to it, and what can be done with it [Hummel et al. 2019].

Data donation arose due to the ubiquity of connected products, services, systems, and ecosystems collecting or producing machine data that reflects the physical and virtual behavior of people. And has been enabled by recent changes in data-sharing and privacy policies such as the GDPR, specifically the right to data portability [GDPR 2018], allowing people to request data collected about them by third parties, reuse and share it [Skatova and Goulding 2019]. Examples of personal data potentially available through data donation include activity data, from mobile phones and wearable devices, reflecting patterns of mobility, isolation, physical activity, and sleep [Strotbaum et al. 2019], transport data, reflecting people's whereabouts and daily context [Skatova et al. 2019], and web logs, reflecting personal interests and concerns [Skatova and Goulding 2019].

So far, data donation, and data donation research, have developed primarily in the context of health research, where data offers a unique opportunity to help others by enabling and advancing health (i.e., research, treatments, medical devices). For example, the Data for Good campaign from PatientsLikeMe ${ }^{1}$, where data donation is presented as an opportunity to help others, better understand personal health, and improve medicine through the exchange of personal data. In this context, researchers have explored what motivates people to participate in data donation and have found that common motives include social duty (i.e., a desire to serve society and give back to the community), self-interest (i.e., a need to gain a personal benefit as a result of data donation), and purpose (i.e., a need to understand the consequences of data donation or what will be done with the data) [Bietz et al. 2019; Skatova and Goulding 2019].

Furthermore, data donation research has also focused on defining and understanding the challenges around data donation [Hummel et al. 2019; Krutzinna et al. 2019; Prainsack 2019]. A key challenge is motivating potential data donors, who are often concerned about their privacy [Hummel et al. 2019]. Especially after cases of data leakage, misuse, or sale, including Cambridge Analytica ${ }^{2}$, the Strava app accidentally revealing the location of U.S. military bases ${ }^{3}$, and Amazon's Alexa sharing private conversations with random contacts ${ }^{4}$, that have led the general public to become increasingly aware of the power and risks of the use and misuse of personal data [Skatova et al. 2019]. The research community argues that for data donation to be effective it has to be conducted in an trusted and ethical way [Krutzinna et al. 2019; Skatova et al. 2019; Wilbanks and Friend 2016]. Which includes, providing the mechanisms for data donors to protect their privacy, understand and access information about how their personal data is used, agree and consent to the use of personal data, and re-evaluate their decisions over time [Hummel et al. 2019; Krutzinna and Floridi 2019].

\footnotetext{
${ }^{1}$ Data for Good Campaign, PatientsLikeMe

${ }^{2}$ Cadwalladr and Graham-Harrison, 50 million Facebook profiles harvested for Cambridge Analytica in major data breach, The Guardian, 2018

${ }^{3}$ Hern, Fitness tracking app Strava gives away location of secret US army bases, The Guardian, 2018

${ }^{4}$ Wolfson, Amazon's Alexa recorded private conversation and sent it to random contact, The Guardian, 2018
} 


\section{DESIGNERLY DATA DONATION}

As described in the previous section, design researchers and practitioners face several challenges to access machine data. First, machine data collection can be burdensome (i.e., expensive, timeconsuming, subject to regulatory frameworks). Second, people might be reluctant to share personal data due to privacy concerns. Third, data often lacks a meaningful contextual grounding. Data donation could address the first of these challenges by enabling design researchers and practitioners to access inexpensive personal machine data with the informed consent of data donors.

However, as currently understood in the medical field, data donation is not sufficient for designerly contexts. Where data is valuable to the extent that it can bring people in and leverage their expertise to contextualize it with their unique sources of knowledge and rich insights from their own experiences [Bogers et al. 2016, 2018; Bourgeois et al. 2014a; Chang et al. 2017]. Unlike the medical field, where large-scale, decontextualized data is highly valuable [Skatova and Goulding 2019; Skatova et al. 2019], designerly contexts require highly contextualized data from different scales, and abstractness [Bogers et al. 2016; Bourgeois et al. 2014a]. For example, an integration of large-scale, decontextualized machine data at the community level, and with highly intimate and detailed contextual insights at the individual level. Where shared sense-making and collaboration with people are essential to transform data into valuable knowledge. Moreover, designerly contexts are not a distant and complex problem, but the path of a shared solution of which the data donor is part [Jung et al. 2017; Sanders 2002]. Therefore, it is necessary to re-conceptualize data donation to fit designerly contexts.

We propose designerly data donation, a participatory approach for machine data collection in-the-wild. Defined as the process by which a person (i.e., data donor) transfers their personal data (i.e., machine data, personal insights, observations), without expecting anything in return, to another person or entity (i.e., data receiver), who will use it in a given context, whereby they become partners actively collaborating in a shared project.

In addition, we propose the potential benefits of designerly data donation around three axes. These were defined through an iterative process where we explored the agents, interactions, and characteristics of (designerly) data donation and clustered them. First, the value gain for both data donors and data receivers. Second, the contextualized data available through designerly data donation. Third, the roles and relationships that derive. In the following sections, we will briefly expand on each theme.

\subsection{Value Gain}

When defining (medical and designerly) data donation, we emphasize that when a data donor transfers their data, they do not expect nor demand anything in return. Donations are not exchanges or directly reciprocal transactions [Prainsack 2019]. However, for them to be successful, something should be given in return. Both data receivers and data donors should gain value from engaging in (medical and designerly) data donation [Krutzinna et al. 2019; Prainsack 2019]. Value gain is straightforward for data receivers as it derive primarily from having affordable and timely access to personal machine data with the consent of data donors. In contrast, data donors could gain value immediately after the exchange (e.g., positive feelings, satisfaction) through insights from data receivers (e.g., understanding data, learning from the process) or in the form of future benefits (e.g., future collaborations, future help) [Bietz et al. 2019; Skatova and Goulding 2019]. Although value gain is fundamental in both medical and designerly data donation, in designerly contexts, the value gain expands as a result of the close collaboration between data donors and data receivers [Bogers et al. 2016; Bourgeois et al. 2014a]. Unlocking access to deeper and more intimate insights for data receivers, and generating direct benefits for data donors, such as access to innovative products and services, or custom design solutions [Bogers et al. 2016; Brown et al. 2016]. Moreover, both parties derive value from their active participation in the design process as collaborators and co-creators of design solutions [Brown et al. 2016].

Fundamental to this collaboration, is the ability to negotiate [Bourgeois et al. 2018; Brown et al. 2016; Mortier et al. 2014]. For data receivers, negotiating includes incentivizing participation, offering potential benefits, and requesting data from different sources and types, as well as different levels of contextual insights [Bietz et al. 2019; Skatova and Goulding 2019]. For data donors, negotiating includes deciding to donate, accepting potential benefits, challenging the decisions that are being made from the data, correcting the assumptions that made through the data, and requesting or providing more information if necessary [Hummel et al. 2019; Prainsack 2019].

The value gain introduces several challenges to designerly data donation. First, guaranteeing that both data donors and data receivers gain value without hampering the process [Brown et al. 2016]. Second, inciting data receivers to comply when the value gain is in the form of future benefits. Third, balancing between collaboration, negotiation, and effectiveness.

\subsection{Contextualized Data}

In the context of (medical and designerly) data donation, data has several characteristics to be highlighted. First, data multiplicity, meaning that data can be in several places at the same time, and therefore data can be donated to, and used by, one or more data receivers at the same time [Prainsack 2019]. Second, data relationality, meaning that data is embedded in social, institutional, political, and economic relations, [Prainsack 2019]. As a result, it often concerns not only individuals but groups and communities. Finally, data sensitivity, meaning that data can contain or disclose highly intimate or sensitive information about certain aspects of personal life [Gabriele and Chiasson 2020].

In designerly data donation, data is not big nor decontextualized. Instead, it is multi-scaled and enriched by the contextual grounding from data donors. As a result, data becomes a participatory design material - designers invite data donors to participate in the design process and co-design solutions. The contextualization of the data is the main differentiator between medical and designerly data donation.

Here, it is critical to ensure that data donors have personal choice and control. The donation of data is an exercise of autonomy, and sovereignty [Hummel et al. 2019; Strotbaum et al. 2019]. As such, data donors must be able to decide whether to donate, what to 
donate, to whom to donate, and under what conditions to donate [Strotbaum et al. 2019]. In the case of designerly data donation, data donors must first actively and knowingly consent to donate their data to a given data receiver in a given context and under certain conditions [Hummel et al. 2019; Jones 2019; Nissembaum 2004] Second, data donors must be able to choose what data to disclose, whether to disclose contextual information that enriches the data, and what attributes and information to provide [Hummel et al. 2019; Nissembaum 2004]. For this, data receivers must inform data donors about the possible implications of sharing certain types of data, and the information that can emerge from combined and highly contextualized data [Goodman 2014; Mortier et al. 2014]. Third, data receivers should support donors in curating their data to refrain from disclosing certain aspects of their personal life, reflected in the data. Finally, data donors should be allowed to reassess their choices, including withdrawing their data when possible [Bourgeois et al. 2018; Mortier et al. 2014].

The contextualized data introduces several challenges to designerly data donation. First, integrating multi-scaled data diversely enriched by contextual insights. Second, supporting data donors - with different backgrounds and data literacy- in exercising choice and control without overloading them. Third, ensuring personal choice and control can conflict with acquiring contextualized quality data at scale and simplifying data collection in designerly contexts. There must be a trade-off between flexibility and functionality.

\subsection{Roles and Relationships}

Cultivating trust is fundamental to both medical and designerly data donation. [Jones 2019]. In the case of medical data donation, data receivers are usually known hospitals, universities or research institutions investigating pressing healthcare issues, likely to inspire trust [Skatova and Goulding 2019; Skatova et al. 2019]. In contrast, data receivers of designerly data donation are design researchers and practitioners responsible for building and cultivating trust in multi-scaled settings. Engaging in designerly data donation enables a closer and intimate relationship between data donors and data receivers. The role of data donors shifts from mere data subjects [Hummel et al. 2019] to active design partners involved in a trusted collaboration towards the co-creation of a designed solution. Nonetheless, clarity and accountability could support the trustworthiness of the designerly data donation process and protect data donors when necessary [Nissembaum 2004].

To ensure a reliable designerly data donation process, data donors must understand from the beginning and throughout the process, how data receivers will use their data and for what purpose [Hummel et al. 2019; Jones 2019]. "Understand" is a keyword here, as it highlights the importance of the information not only be made accessible to data donors in a transparent way but also clear and legible [Brown et al. 2016; Mortier et al. 2014]. Data receivers have the responsibility to establish and maintain open communication channels to keep data donors up-to-date with regards to the analyses performed on their data and the information derived from it [Diethei et al. 2021; Jones 2019].

The changing roles and relationships resulting from designerly data donation are precious for co-designing data-centric design solutions. However, they introduce a few challenges to the designerly data donation process. First, data receivers bear an ethical responsibility and are accountable to data donors [Bietz et al. 2019; Strotbaum et al. 2019]. However, this is far from a legal oversight or regulatory framework. Second, data receivers ought to balance clarity and security. Third, there is a need to ensure that the data is used within the context that it was shared for and not further [Jones 2019; Wilbanks and Friend 2016].

In this section, we introduced designerly data donation as an effective and ethical way to enable data-centric design. For this, we defined the core issues of designerly data donation, emphasizing the similarities and differences from medical data donation. In addition, we highlighted how designerly data donation could enable designers to overcome some of the challenges they currently face with machine data collection (i.e., burden, people's reluctance to share due to privacy concerns, lack of contextual grounding). Furthermore, we briefly introduce some of the challenges of designerly data donation, such as integration with existing regulatory frameworks (e.g., GDPR) and compliance, ethical oversight, integration of data and flexibility.

\section{FUTURE RESEARCH}

Through the concept of designerly data donation, we open an avenue to empower designers with emerging machine data opportunities. Rather than looking at opposing qualities such as anonymity and unidirectional data collection, we propose a focus on active participation in win-win data collaboration. We make a call for further investigation into the theoretical and practical challenges of designerly data donation, including integration with existing regulatory frameworks (e.g., GDPR) and compliance, ethical oversight, integration of data and flexibility.

First, it remains unclear how design researchers and practitioners would leverage designerly data donation in practice. It is fundamental to understand the perspectives of potential data receivers from research and industry. How does designerly data donation fit their current processes and practices? Second, collaborating with design researchers and practitioners may bring clear and meaningful purposes for data gathering that could allow the exploration of the attitudes and perspectives of individuals and communities about personal data donation. What are the privacy expectations that govern designerly data donation? What contextual and personal factors influence these expectations the most? Third, future work should inquire about effective ways to incentivize and encourage participation as well as long term engagement across a diverse range of individuals to avoid inadvertently excluding people from co-design processes. What are the factors that influence the success of a designerly data donation request? How can these be used to incentivize participation? Finally, future work at the intersection of HCI, UbiComp, and design, should investigate the practical implementation challenges of designerly data donation. How can we design a process for effective data donation, accounting for highly dynamic data sets? 


\section{CONCLUSION}

For designers and researchers, in-the-wild research means having access to rich spatiotemporal insights reflecting user's characteristics, behaviours, and needs. For example, in the context of blended experiences, where a remote understanding of how people perceive, experience, and inhabit digital and physical spaces is needed. However, designerly contexts require contextualized and meaningful data, and collecting it in-the-wild involves a great effort. In addition, ethical implications need to be considered. In this paper, we propose designerly data donation, a participatory approach for data collection in-the-wild, as an effective and ethical way to enable data-centric design processes. We introduce the potential benefits of designerly data donation around three axes: value gain, data contextualization, and roles and relationships.

\section{REFERENCES}

Matthew Bietz, Kevin Patrick, and Cinnamon Bloss. 2019. Data Donation as a Model for Citizen Science Health Research. Citizen Science: Theory and Practice 4, 1 (2019), 1-11. https://doi.org/10.5334/cstp. 178

Sander Bogers, Joep Frens, Janne Van Kollenburg, Eva Deckers, and Caroline Hummels. 2016. Connected baby bottle: A design case study towards a framework for dataenabled design. In DIS 2016 - Proceedings of the 2016 ACM Conference on Designing Interactive Systems: Fuse. Association for Computing Machinery, Inc, New York, NY, USA, 301-311. https://doi.org/10.1145/2901790.2901855

Sander Bogers, Janne Van Kollenburg, Eva Deckers, Joep Frens, and Caroline Hummels 2018. A situated exploration of designing for personal health ecosystems through data-enabled design. In DIS 2018 - Proceedings of the 2018 Designing Interactive Systems Conference. Association for Computing Machinery, Inc, New York, NY, USA, 109-120. https://doi.org/10.1145/3196709.3196769

Tobias Bornakke and Brian L. Due. 2018. Big-Thick Blending: A method for mixing analytical insights from big and thick data sources. Big Data and Society 5, 1 (2018), 1-16. https://doi.org/10.1177/2053951718765026

Jacky Bourgeois, Gerd Kortuem, and Fahim Kawsar. 2018. Trusted and GDPR-compliant research with the internet of things. In ACM International Conference Proceeding Series. Association for Computing Machinery, New York, NY, USA, 1-8. https: //doi.org/10.1145/3277593.3277604

Jacky Bourgeois, Janet Van Der Linden, Gerd Kortuem, Blaine A. Price, and Christopher Rimmer. 2014a. Conversations with my washing machine: An in-the-wild study of demand shifting with self-generated energy. In UbiComp 2014 - Proceedings of the 2014 ACM International foint Conference on Pervasive and Ubiquitous Computing. Association for Computing Machinery, New York, NY, USA, 459-470. https://doi. org/10.1145/2632048.2632106

J. Bourgeois, J. Van Der Linden, G. Kortuem, B. A. Price, and C. Rimmer. 2014b. Using participatory data analysis to understand social constraints and opportunities of electricity demand-shifting. In ICT for Sustainability 2014, ICT4S 2014. Atlantis Press, Stockholm, Sweden, 392-401. https://doi.org/10.2991/ict4s-14.2014.49

Barry Brown, Alexandra Weilenmann, Donald Mcmillan, and Airi Lampinen. 2016. Five Provocations for Ethical HCI R esearch. In Proceedings of the 2016 CHI Conference on Human Factors in Computing Systems. Association for Computing Machinery, New York, NY, USA, 852-863. https://doi.org/10.1145/2858036.2858313

Alan Chamberlain and Andy Crabtree. 2020. Research 'In the Wild'. In Into the Wild: Beyond the Design Research Lab. Studies in Applied Philosophy, Epistemology, and Rational Ethics., A. Chamberlain and A. Crabtree (eds.) (Ed.). Springer Nature Switzerland AG, Nottingham, Nottingham, UK, 1-6. https://doi.org/10.1007/9783-030-18020-1

Wen Wei Chang, Elisa Giaccardi, Lin Lin Chen, and Rung Huei Liang. 2017. "Interview with things:" A first-thing perspective to understand the scooter's everyday sociomaterial network in Taiwan. DIS 2017 - Proceedings of the 2017 ACM Conference on Designing Interactive Systems 1 (2017), 1001-1012. https://doi.org/10.1145/3064663. 3064717

Elizabeth F. Churchill. 2018. Data, design, and ethnography. Interactions 25, 1 (2018), 22-23. https://doi.org/10.1145/3172893

Andy Crabtree, Peter Tolmie, and Alan Chamberlain. 2020. "Research in the Wild" Approaches to Understanding the Unremarkable as a Resource for Design. Studies in Applied Philosophy, Epistemology and Rational Ethics 48 (2020), 31-53. https: //doi.org/10.1007/978-3-030-18020-1\{_\}3

Daniel Diethei, Jasmin Niess, Carolin Stellmacher, Evropi Stefanidi, and Johannes Schöning. 2021. Sharing Heartbeats: Motivations of Citizen Scientists in Times of Crises. Proceedings of the 2021 CHI Conference on Human Factors in Computing Systems 2020, April (2021), 15. https://doi.org/10.1145/3411764.3445665
Graham Dove, Kim Halskov, Jodi Forlizzi, and John Zimmerman. 2017. UX design innovation: Challenges for working with machine learning as a design material. Conference on Human Factors in Computing Systems - Proceedings 2017-May (2017), 278-288. https://doi.org/10.1145/3025453.3025739

Sandra Gabriele and Sonia Chiasson. 2020. Understanding Fitness Tracker Users' Security and Privacy Knowledge, Attitudes and Behaviours. In Conference on Human Factors in Computing Systems - Proceedings. Association for Computing Machinery, New York, NY, USA, 1-12. https://doi.org/10.1145/3313831.3376651

GDPR. 2018. General Data Protection Regulation. , 88 pages. https: //ec.europa.eu/commission/sites/beta-political/files/data-protection-factsheetchanges_en.pdf\%7D

Elisa Giaccardi, Nazli Cila, Chris Speed, and Melissa Caldwell. 2016. Thing ethnography: Doing design research with non-humans. In DIS 2016 - Proceedings of the 2016 ACM Conference on Designing Interactive Systems: Fuse. Association for Computing Machinery, New York, NY, USA, 377-387. https://doi.org/10.1145/2901790.2901905

Elizabeth Goodman. 2014. Design and ethics in the era of big data. Interactions 21, 3 (2014), 22-24. https://doi.org/10.1145/2598902

Katerina Gorkovenko, Daniel J Burnett, James K Thorp, Daniel Richards, and Dave Murray-Rust. 2020. Exploring the Future of Data-Driven Product Design. In Conference on Human Factors in Computing Systems - Proceedings. Association for Computing Machinery, New York, NY, USA, 1-14. https://doi.org/10.1145/3313831.3376560

Patrik Hummel, Matthias Braun, and Peter Dabrock. 2019. Data Donations as Exercises of Sovereignty. Vol. 137. Springer International Publishing, Cham. 23-54 pages. https://doi.org/10.1007/978-3-030-04363-6\{_\}3

Hilary Hutchinson, Wendy Mackay, Bosse Westerlund, Benjamin B. Bederson, Allison Druin, Catherine Plaisant, Michel Beaudouin-Lafon, Stéphane Conversy, Helen Evans, Heiko Hansen, Nicolas Roussel, Björn Eiderbäck, Sinna Lindquist, and Yngve Sundblad. 2003. Technology probes: Inspiring design for and with families. In Conference on Human Factors in Computing Systems - Proceedings. Association for Computing Machinery, New York, NY, USA, 17-24. https://doi.org/10.1145/ 642611.642616

Kerina H. Jones. 2019. Incongruities and Dilemmas in Data Donation: Juggling Our 1s and 0s. Philosophical Studies Series 137 (2019), 75-93. https://doi.org/10.1007/9783-030-04363-6\{_\}5

Young Wook Jung, Youn Kyung Lim, and Myung Suk Kim. 2017. Possibilities and limitations of online document tools for design collaboration: The case of Google Docs. In Proceedings of the ACM Conference on Computer Supported Cooperative Work, CSCW. Association for Computing Machinery, New York, New York, USA, 1096-1108. https://doi.org/10.1145/2998181.2998297

Jenny Krutzinna and Luciano Floridi. 2019. Ethical Medical Data Donation: A Pressing Issue. Philosophical Studies Series 137 (2019), 1-6. https://doi.org/10.1007/978-3030-04363-6\{_\}1

Jenny Krutzinna, Mariarosaria Taddeo, and Luciano Floridi. 2019. An Ethical Code for Posthumous Medical Data Donation. Philosophical Studies Series 137 (2019), 181-195. https://doi.org/10.1007/978-3-030-04363-6\{_\}12

Richard Mortier, Hamed Haddadi, Tristan Henderson, Derek McAuley, and Jon Crowcroft. 2014. Human-Data Interaction: The Human Face of the Data-Driven Society. SSRN Electronic fournal 10 (2014), 1-11. https://doi.org/10.2139/ssrn.2508051

Helen Nissembaum. 2004. Privacy as Contextual Integrity. Washington Law Review 79, 1 (2004), 101-139.

Barbara Prainsack. 2019. Data Donation: How to Resist the iLeviathan. Philosophical Studies Series 137 (2019), 9-22. https://doi.org/10.1007/978-3-030-04363-6\{_\}2

Tony Salvador, Genevive Bell, and Ken Anderson. 2020. Design Ethnography. Research Methods for Interior Design 10, 617 (2020), 30-47. https://doi.org/10.4324/ 9780429029325-3

Elizabeth Sanders. 2002. From user-centered to participatory design approaches. Design and the Social Sciences 04 (2002), 1-7. https://doi.org/10.1201/9780203301302.ch1

Anya Skatova and James Goulding. 2019. Psychology of personal data donation. PLoS ONE 14, 11 (2019), 1-20. https://doi.org/10.1371/journal.pone.0224240

Anya Skatova, Kate Shiells, and Andy Boyd. 2019. Attitudes towards transactional data donation and linkage in a longitudinal population study: Evidence from the Avon Longitudinal Study of Parents and Children. Wellcome Open Research 4 (2019), 1-16. https://doi.org/10.12688/wellcomeopenres.15557.1

Chris Speed and Jon Oberlander. 2016. Designing from, with and by Data: Introducing the ablative framework. In Proceedings of DRS 2016 International Conference: Future-Focused Thinking. Design Research Society, Brighton, UK, 2991-3004.

Veronika Strotbaum, Monika Pobiruchin, Björn Schreiweis, Martin Wiesner, and Brigitte Strahwald. 2019. Your data is gold - Data donation for better healthcare? IT - Information Technology 61, 5-6 (2019), 219-229. https://doi.org/10.1515/itit2019-0024

Niels Van Berkel, Denzil Ferreira, and Vassilis Kostakos. 2017. The experience sampling method on mobile devices. Comput. Surveys 50, 6 (2017), 40. https://doi.org/10. $1145 / 3123988$

Roberto Verganti, Luca Vendraminelli, and Marco Iansiti. 2020. Innovation and Design in the Age of Artificial Intelligence. Fournal of Product Innovation Management 37, 3 (2020), 212-227. https://doi.org/10.1111/jpim.12523

John Wilbanks and Stephen H. Friend. 2016. First, design for data sharing. Nature Biotechnology 34, 4 (2016), 377-379. https://doi.org/10.1038/nbt.3516 
Peter Worthy, Ben Matthews, and Stephen Viller. 2016. Trust me: Doubts and concerns living with the internet of things. In DIS 2016 - Proceedings of the 2016 ACM Conference on Designing Interactive Systems: Fuse. Association for Computing Machinery,
New York, NY, USA, 427-434. https://doi.org/10.1145/2901790.2901890 